\title{
MÉTODOS ULTRAVIOLETA SELECTIVO Y DE REDUCCIÓN CON HIDRACINA EN LA DETERMINACIÓN DEL IÓN NITRATO AN AGUAS SUBTERRANEAS
}

\author{
Roberto F. Belgrano, Viviana Colasurdo y Oscar A. Díaz* \\ Facultad de Ingeniería, Universidad Nacional del Centro de la Provincia de Buenos Aires, Av. Del Valle 5737, B7400JWU Olavarría \\ - Argentina
}

Recebido em 19/11/02; aceito em 26/2/03

\begin{abstract}
SELECTIVE UV METHOD AND REDUCTION WITH HIDRAZINE METHOD IN THE DETERMINATION OF NITRATE ION IN GROUNDWATER. In this study, a comparison of two methods, the Selective UV Spectrophotometrical Method and Reduction with Hidrazine Method, for the determination of Nitrate ion in groundwater, was carried out. For this purpose, the results from all the drinking water collectings of the city of Olavarría, Argentina, were employed. Both methods present significant different means, but they don't present significant differences in their variances.
\end{abstract}

Keywords: Hidrazine; Ultraviolet; Nitrate.

\section{INTRODUCCIÓN}

El ion nitrato es una especie química cada vez más frecuente en las aguas superficiales y subterráneas. Las causas y consecuencias de la contaminación de acuíferos por la presencia de este ion, es un hecho en creciente estudio desde hace varios años ${ }^{1-5}$.

A menos que se tomen medidas para limitar la magnitud y velocidad del aporte de nitratos a la masa acuosa del planeta, los problemas que hasta ahora han sido locales o regionales, podrían alcanzar dimensiones continentales o hasta globales, con una contaminación general de las aguas dulces y marinas.

Si los nitratos dejaran de utilizarse como fertilizantes, millones de personas morirían de hambre. Por el contrario si siguen siendo usados al ritmo actual, en menos de 20 años, la contaminación del medio ambiente se habrá generalizado ${ }^{6}$. En este sentido hay que recordar que en algunos países se están consumiendo aguas obtenidas por mezclas de agua de alta calidad con otra de más alto nivel de nitratos, para así obtener un producto aceptable ${ }^{1}$.

Los consumos de alimentos o aguas con altos contenidos de nitratos conllevan peligros a la salud humana. La conocida methemoglobinemia es provocada por la sobredosis de nitritos, que en el caso de consumo de aguas de alto tenor de nitratos son generados en el estómago. Por otra parte es bien sabido que por múltiples y muy versátiles mecanismos, los nitritos en medios diferentes y en presencia de substratos portadores del grupo amino, dan lugar a la formación de nitroso compuestos especies de capacidad cancerígena probada ${ }^{6}$.

Para evitar los efectos adversos del nitrato sobre la salud humana, la concentración del mismo a sido limitada en aguas de bebida a $45 \mathrm{mgNO}_{3}^{-} / \mathrm{L}$ en Estados Unidos, $55 \mathrm{mgNO}_{3}^{-} / \mathrm{L}^{-}$en Europa ${ }^{7}$ y 45 $\mathrm{mgNO}_{3} / \mathrm{L}$ en Argentina ${ }^{8}$.

En nuestro país casi la cuarta parte de los abastecimientos de agua potable, lo hacen a través de la extracción de aguas subterráneas9. En la ciudad de Olavarría (Provincia de Buenos Aires) el abastecimiento de agua potable se realiza totalmente a partir de aguas subterráneas, estando a cargo del servicio la Cooperativa Limitada de Consumo de Electricidad y Servicios Anexos de Olavarría (Coopelectric) que opera una batería de captaciones en el radio urbano.

\footnotetext{
*e-mail: odiaz@fio.unicen.edu.ar
}

Desde el mes de junio del año 1998, los autores realizan el monitoreo del ion nitrato en la totalidad de los pozos que el servicio posee en explotación.

La determinación de nitratos en aguas es difícil dado los procedimientos complejos con los que se cuentan, la gran posibilidad de encontrar sustancias interferentes y los rangos de concentración limitados que presentan las diferentes técnicas.

Entre los métodos que aparecen en la bibliografía para la cuantificación de nitratos en aguas, se pueden $\operatorname{citar}^{10}$ : Cromatografía iónica, método espectrofotométrico ultravioleta selectivo, método del electrodo de nitrato, método de reducción de cadmio, método del cloruro titanoso, método automatizado de reducción de hidracina, método automático de reducción de cadmio.

En el presente trabajo se llevó a cabo la comparación de dos métodos de determinación de ion nitrato en muestras de aguas: el Método Espectrofotométrico Ultravioleta Selectivo (MUV) y el Método de la Reducción con Hidracina (MRH).

Las técnicas comparadas se diferencian en su especificidad, ya que el MUV, no es un método específico para la determinación de ion nitrato en agua y debe ser utilizado con los recaudos necesarios.

Se emplearon los resultados obtenidos del monitoreo de las 26 captaciones de agua potable de la ciudad de Olavarría, Provincia de Buenos Aires, Argentina. Los autores utilizaron las mismas captaciones para comparar otros métodos ${ }^{11}$.

\section{PARTE EXPERIMENTAL}

Durante cinco meses se efectuó un muestreo cada treinta días aproximadamente. Las muestras registradas fueron obtenidas en 26 estaciones distintas. Esta frecuencia de muestreo arroja una población de 126 valores.

Cada una de las muestras fue preservada en refrigerador y procesada dentro de las $24 \mathrm{~h}$ de su extracción, aunque algunos autores manifiestan haber realizado las determinaciones dentro de los 14 días sin variaciones significativas ${ }^{2}$.

En ambos métodos se trabajó con un espectrofotómetro METROLAB 1700 UV - Visible.

El MUV, permite cuantificar nitratos en forma rápida. Una vez tomada la muestra y antes de las $24 \mathrm{~h}$, se determina la absorbancia a $220 \mathrm{~nm}$, previo agregado de $\mathrm{HCl} 1 \mathrm{M}$ para eliminar interferencias. 
Dado que la materia orgánica puede absorber también a 220 nm, se realiza otra medición a $275 \mathrm{~nm}$, para corregir el valor de la lectura correspondiente a nitrato.

Este método presenta la dificultad de no ser adecuado para el estudio de aguas contaminadas, es decir sólo es válida su aplicación para muestras de agua con bajo contenido en materia orgánica, característica que cumplen todas las muestras analizadas en este trabajo.

Se preparó una curva de calibración con un patrón de nitrato de potasio que abarcara un rango de 0 a $7 \mathrm{mgN}-\mathrm{NO} 3-/ 1$, que arrojó un coeficiente de regresión $\left(\mathrm{R}^{2}\right)$ de 0.997 .

El MRH se basa en la reducción de nitrato a nitrito, empleando como agente reductor al sulfato de hidracina. Luego el nitrito se determina espectrofotométricamente a $540 \mathrm{~nm}$, longitud de onda que es absorbida por el colorante azo, que se forma por diazotación con sulfanilamida y apareamiento con diclorhidrato de N-(1-naftil)etilendiamina.

Para realizar la curva de calibración se usaron los mismos patrones utilizados en el MUV. El ajuste de dicha curva arrojó un coeficiente de regresión $\left(\mathrm{R}^{2}\right)$ de 0.995 .

El rango de aplicación de este método es de 0.01 a $10 \mathrm{mg}$ $\mathrm{N}\left(\mathrm{NO}_{3}^{-}\right) / \mathrm{L}$.

Los resultados de las determinaciones realizadas se muestran en las Tablas 1 y 2.

\section{RESULTADOS Y DISCUSION}

A efectos de comparar y caracterizar ambos métodos, se realizaron análisis estadísticos e inferenciales. Los datos fueron procesados con el Software Microsoft Excel 97.

Tabla 1. Concentración de ion nitrato, en $\mathrm{mg} / \mathrm{L}$, obtenidos por el método de la radiación ultravioleta

\begin{tabular}{cccccc}
\hline Pozo & $6 / 03 / 02$ & $10 / 04 / 02$ & $11 / 06 / 02$ & $27 / 07 / 02$ & $3 / 09 / 02$ \\
\hline 2 & 13,0 & 11,2 & 11,4 & 11,4 & 12,8 \\
3 & 13,1 & 14,3 & 14,3 & 10,0 & 13,7 \\
4 & 13,4 & 16,7 & 15,6 & 16,7 & 13,3 \\
5 & 16,5 & 14,8 & 14,1 & 15,4 & 14,2 \\
6 & 20,8 & 21,6 & 20,8 & 21,7 & 21,2 \\
7 & 20,6 & 24,2 & 22,4 & 24,6 & 20,4 \\
8 & 18,3 & 17,2 & 21,7 & 31,1 & 30,0 \\
9 & 36,5 & 55,0 & 31,8 & 59,2 & 37,3 \\
10 & 14,8 & 15,4 & 13,9 & 15,8 & 15,3 \\
11 & 20,5 & 21,3 & 21,5 & 22,9 & 22,2 \\
12 & 14,8 & 15,8 & 14,7 & 15,4 & 15,2 \\
13 & 15,7 & 14,3 & 15,8 & 15,1 & 13,1 \\
14 & & 13,6 & 13,1 & 14,1 & 13,6 \\
15 & 17,9 & & 16,6 & & 16,8 \\
16 & 23,8 & 24,0 & 24,4 & 25,1 & 24,4 \\
17 & 14,4 & 15,0 & 14,1 & 14,2 & 14,9 \\
18 & 17,4 & & 16,3 & 16,6 & 16,6 \\
19 & 14,4 & 14,3 & 14,0 & 13,8 & 14,5 \\
20 & 24,1 & 18,0 & 19,2 & 15,4 & 20,3 \\
21 & 42,6 & 48,0 & 46,1 & 48,3 & 41,9 \\
22 & 14,9 & 15,1 & 14,4 & 14,0 & 12,8 \\
31 & 55,9 & 57,0 & 55,4 & 57,5 & 67,4 \\
32 & 57,3 & 58,0 & 55,0 & 62,5 & 57,2 \\
33 & 26,8 & 27,6 & 28,3 & 29,5 & 27,7 \\
34 & 22,5 & 23,3 & 23,6 & 25,1 & 23,5 \\
PIO & 10,6 & 10,4 & 10,1 & 10,8 & 10,0 \\
\hline
\end{tabular}

*Los casilleros en blanco corresponden a datos no reportados, debido en que las bombas se encontraban fuera de servicio al momento de la toma de muestra
Tabla 2. Concentración de ion nitrato, en $\mathrm{mg} / \mathrm{L}$, obtenidos por el método de la reducción con hidracina

\begin{tabular}{cccccc}
\hline Pozo & $6 / 03 / 02$ & $10 / 04 / 02$ & $11 / 06 / 02$ & $27 / 07 / 02$ & $3 / 09 / 02$ \\
\hline 2 & 16,0 & 14,8 & 10,3 & 12,6 & 15,2 \\
3 & 11,5 & 14,8 & 12,5 & 13,4 & 13,9 \\
4 & 10,2 & 19,5 & 16,8 & 20,1 & 18,1 \\
5 & 18,1 & 16,8 & 11,5 & 19,0 & 16,0 \\
6 & 18,9 & 25,4 & 23,6 & 26,5 & 25,3 \\
7 & 17,9 & 28,7 & 21,8 & 30,1 & 24,2 \\
8 & 16,9 & 18,7 & 17,9 & 34,3 & 32,1 \\
9 & 38,2 & 57,6 & 35,1 & 62,5 & 41,3 \\
10 & 15,5 & 14,8 & 19,3 & 19,0 & 17,3 \\
11 & 18,7 & 26,5 & 25 & 24,5 & 25,8 \\
12 & 11,4 & 17,8 & 16,3 & 19,1 & 19,7 \\
13 & 14,7 & 16,0 & 18,5 & 18,5 & 16,2 \\
14 & & 16,2 & 14,0 & 18,6 & 16,3 \\
15 & 15,3 & & 15,2 & & 15,7 \\
16 & 19,7 & 28,4 & 26,5 & 26,4 & 24,3 \\
17 & 16,1 & 14,9 & 11,2 & 15,8 & 20,0 \\
18 & 15,9 & & 15,7 & 22,1 & 17,2 \\
19 & 20,4 & 15,1 & 19 & 15,8 & 17,9 \\
20 & 28,7 & 18,3 & 22,5 & 21,3 & 22,7 \\
21 & 44,6 & 52,2 & 48,2 & 52,4 & 45,6 \\
22 & 13,7 & 19,2 & 12,6 & 19,2 & 17,6 \\
31 & 52,3 & 55,2 & 55,8 & 60,2 & 64,2 \\
32 & 55,4 & 60,6 & 49,6 & 63,1 & 55,3 \\
33 & 23,7 & 30,2 & 25,6 & 34,0 & 29,4 \\
34 & 19,9 & 24,3 & 22,7 & 27,5 & 24,1 \\
PIO & 11,3 & 12,3 & 7,9 & 13,8 & 12,5 \\
\hline
\end{tabular}

*Los casilleros en blanco corresponden a datos no reportados, debido en que las bombas se encontraban fuera de servicio al momento de la toma de muestra

En la Tabla 3, se muestran los valores de las variables descriptivas calculadas para ambos métodos.

El nivel de concentración media de nitratos por ambas técnicas arrojó diferencias significativas, por defecto, con el valor límite superior de $45 \mathrm{mg} / \mathrm{L}$, establecido por el Código Alimentario Argentino, para aguas de bebida.

Los coeficientes de variabilidad (desviación estándar/media) para ambos métodos, son semejantes, 0,5587 para el MRH, y 0,5998 para el MUV. El MRH se presenta un poco más estable a las diferencias de concentración.

Se aplicó la Prueba t por parejas, o sea que se analizó la diferencia entre cada par de determinaciones dadas por los dos métodos ${ }^{12,13}$. Los resultados obtenidos para esta prueba se muestran en la Tabla 4.

Tabla 3. Variables estadísticas descriptivas para ambos métodos

\begin{tabular}{lll}
\hline & MUV & MRH \\
\hline Media & 22,95 & 24,41 \\
Error típico & 1,23 & 1,22 \\
Mediana & 16,70 & 19,05 \\
Moda & 14,30 & 16,00 \\
Desviación estándar & 13,77 & 13,64 \\
Varianza de la muestra & 189,50 & 186,05 \\
Curtosis & 1,95 & 1,49 \\
Coeficiente de asimetría & 1,73 & 1,57 \\
Rango & 57,40 & 56,30 \\
Mínimo & 10,00 & 7,90 \\
Máximo & 67,40 & 64,20 \\
Suma & 2891,80 & 3076,10 \\
Cuenta & 126,00 & 126,00 \\
\hline
\end{tabular}


Tabla 4. Prueba t para media de dos muestras emparejadas

\begin{tabular}{lll}
\hline & MUV & MRH \\
\hline Media & 22,95 & 24,41 \\
Varianza & 189,50 & 186,05 \\
Observaciones & 126 & 126 \\
Coeficiente de correlación de Pearson & 0,98 & \\
Diferencia hipotética de las medias & 0 & \\
Grados de libertad & 125 & \\
Estadístico t & $-6,23$ & \\
$\mathrm{P}(\mathrm{T}<=\mathrm{t})$ una cola & $3,3310^{-9}$ & \\
Valor crítico de t (una cola) & 1,66 & \\
$\mathrm{P}(\mathrm{T}<=\mathrm{t})$ dos colas & $6,6710^{-9}$ & \\
Valor crítico de $\mathrm{t}$ (dos colas) & 1,98 & \\
\hline
\end{tabular}

El rechazo de la hipótesis nula, indica que ambos métodos presentan valores para las concentraciones medias de nitratos, que difieren significativamente entre sí, para un nivel de confianza del $95 \%$.

Para comparar la precisión de ambos métodos, se llevó a cabo la Prueba $\mathrm{F}^{12,13}$. Los resultados obtenidos se muestran en la Tabla 5.

Tabla 5. Prueba F para varianzas de dos muestras

\begin{tabular}{lll}
\hline & MUV & MRH \\
\hline Media & 22,95 & 24,41 \\
Varianza & 189,50 & 186,05 \\
Observaciones & 126 & 126 \\
Grados de libertad & 125 & 125 \\
$\mathrm{~F}$ & 1,02 & \\
$\mathrm{P}(\mathrm{F}<=$ f) una cola & 0,46 & \\
Valor crítico para F (una cola) & 1,34 & \\
\hline
\end{tabular}

Se puede observar, que no hay diferencias significativas entre las dos varianzas, a un nivel de confianza del $95 \%$.

\section{CONCLUSIONES}

Para la determinación de ion nitrato en aguas subterráneas, el método espectrofotométrico ultravioleta selectivo presenta como características principales la baja demanda de tiempo y reactivos necesarios y su fácil ejecución. Comparativamente, esta es una ventaja frente al método de la reducción con hidracina.

$\mathrm{Si}$ bien ambos métodos proporcionan valores para las concentraciones medias de este ion que difieren significativamente, se caracterizan por ser igualmente precisos.

\section{REFERENCIAS}

1. Ronen, D.; Kanfi, Y.; Magaritz, M.; Water Res. 1983, 17, 1499.

2. Kanfi, Y.; Ronen, D.; Magaritz, M.; Journal of Hydrology 1983, 66, 331.

3. Addiscott, T.; Whitmore, A.; Soil Use and Management 1991, 7, 94.

4. Addiscot, T.; Wagenet, R.; Journal of Soil Science 1985, 29, 305.

5. Ronen, D.; Belgiun $1^{\text {st }}$ Symposiun on Groundwater Quality Control Management, Brussels, Belgiun, 1974.

6. Casado, J.; Resúmenes de Conferencias y Comunicaciones de IX Encontro Galego Portugues de Química, Lugo, Galicia, 1995.

7. Glass, C.; Silverstein, J.; Water Res. 1999, 33, 223.

8. Código Alimentario Argentino, de la Canal y Asociados, Argentina, 1999.

9. Conapa, Informe Nacional a la Conferencia sobre Medio Ambiente y Desarrollo de las Naciones Unidas, Comisión Nacional de Política Ambiental (Secretaria General Presidencia de la Nación), Argentina, 1991.

10. APHA-AWWA-WPCF; Métodos Normalizados para el Análisis de Aguas Potables y Residuales, Ed. Diaz de Santos, S. A.: Madrid, 1992.

11. Colasurdo, V.; Sager, E.; Cocconi, M.; Díaz, O.; Afinidad 2001, LVIII, 329.

12. Miller, J. C.; Miller, J. N.; Estadística para Química Analítica, AddisonWesley Iberoamericana S.A.: Delaware, 1993

13. Blanch, N.; Joekes, S.; Estadística aplicada a la investigación, 6ta. ed., Universidad Nacional de Córdoba: Argentina, 1997. 